\title{
Treatment of knee osteoarthritis
} with intra-articular injection of allogeneic adipose-derived stem cells (ADSCS) ELIXCYTE ${ }^{\circledR}$ : a phase I/II, randomized, active-control, single-blind, multiple-center clinical trial

Cheng-Fong Chen ${ }^{1,2+}{ }^{\text {, Chih-Chien Hu } u^{3,4,5 \dagger} \text {, Chen-Te Wu }{ }^{6} \text {, Hung-Ta H. Wu }{ }^{7,8} \text {, Chun-Shin Chang }}{ }^{9,10}$, Yi-Pei Hung ${ }^{11}$, Chia-Chu Tsai ${ }^{11}$ and Yuhan Chang $3,4,5^{*}$ (D)

\begin{abstract}
Objective: To evaluate the safety and efficacy of intra-articular (IA) injection of allogeneic adipose-derived stem cells (ADSCS) ELIXCYTE ${ }^{\circledR}$ for knee osteoarthritis.

Methods: This was a patient-blind, randomized, active-control trial consisted of 4 arms including hyaluronic acid (HA) control and 3 ELIXCYTE ${ }^{\circledR}$ doses. A total of 64 subjects were screened, and 57 subjects were randomized. The primary endpoints included the changes from baseline to post-treatment visit of Western Ontario and McMaster Universities Osteoarthritis Index (WOMAC) pain score at Week 24 and the incidence of adverse events (AEs) and serious adverse events (SAEs).
\end{abstract}

Results: No ELIXCYTE ${ }^{\circledR}$-related serious adverse events were reported during 96 weeks of follow-up and no suspected unexpected serious adverse reaction (SUSAR) or death was reported. The changes of the primary endpoint, WOMAC pain score at Week 24, showed significant differences in all ELIXCYTE ${ }^{\circledR}$ groups, as well as in HA groups between posttreatment visit and baseline. The ELIXCYTE ${ }^{\circledR}$ groups revealed significant decreases at Week 4 compared to HA group in WOMAC total scores, stiffness scores, functional limitation scores suggested the potential of ELIXCYTE ${ }^{\circledR}$ in earlier onset compared to those from HA. The significant differences of visual analog scale (VAS) pain score and Knee Society Clinical Rating System (KSCRS) functional activities score at Week 48 after ELIXCYTE ${ }^{\circledR}$ administration suggested the potential of ELIXCYTE ${ }^{\circledR}$ in the longer duration of the effectiveness compared to HA group.

Conclusions: ELIXCYTE ${ }^{\circledR}$ for knee osteoarthritis treatment was effective, safe, and well-tolerated. The efficacy results were showed that ELIXCYTE ${ }^{\circledR}$ conferred the earlier onset of reductions in pain scores and improvements in functional scores than HA group.

\footnotetext{
*Correspondence: yhchang@cgmh.org.tw

${ }^{\dagger}$ Cheng-Fong Chen and Chih-Chien Hu have been contributed equally to this work

${ }^{3}$ Division of Joint Reconstruction, Department of Orthopedic Surgery,

Chang Gung Memorial Hospital, Linkou, Taiwan

Full list of author information is available at the end of the article
}

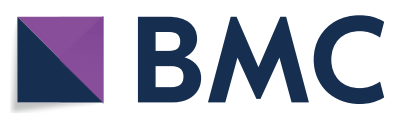

(c) The Author(s) 2021. Open Access This article is licensed under a Creative Commons Attribution 4.0 International License, which permits use, sharing, adaptation, distribution and reproduction in any medium or format, as long as you give appropriate credit to the original author(s) and the source, provide a link to the Creative Commons licence, and indicate if changes were made. The images or other third party material in this article are included in the article's Creative Commons licence, unless indicated otherwise in a credit line to the material. If material is not included in the article's Creative Commons licence and your intended use is not permitted by statutory regulation or exceeds the permitted use, you will need to obtain permission directly from the copyright holder. To view a copy of this licence, visit http://creativecommons.org/licenses/by/4.0/. The Creative Commons Public Domain Dedication waiver (http://creativecommons.org/publicdomain/zero/1.0/) applies to the data made available in this article, unless otherwise stated in a credit line to the data. 
Trial registration: ClinicalTrials.gov Identifier: NCT02784964. Registered 16 May, 2016—Retrospectively registered, https://clinicaltrials.gov/ct2/show/NCT02784964

Keywords: Knee osteoarthritis, Adipose tissue-derived stem cells, ADSCs, ELIXCYTE ${ }^{\circledR}$, Hyaluronic acid, HA, WOMAC, VAS, KSCRS

\section{Background}

Knee osteoarthritis (KOA) is a chronic musculoskeletal disease that affects $7 \%$ of the global population, more than 500 million people, and has been recognized as the 15 th highest cause of years lived with disability (YLDs) worldwide [1]. Current managements of KOA are aimed at pain reduction and symptom control rather than disease modification. Nonsteroidal anti-inflammatory drugs (NSAIDs), acetaminophen, and corticosteroids are generally used, and patients are unsatisfied with their limited analgesic, short-term efficacy, and potential risk of gastrointestinal and cardiovascular disorder [2, 3]. Hyaluronic acid (HA) is a natural component that seems to be attributable to KOA therapy via its viscosupplementation properties. Even though the use of intra-articular HA has raised no major safety concerns so far, the efficacy is still controversial [4].

Novel findings on the mechanisms underlying the development of KOA promote the research of potential disease-modifying osteoarthritis drugs (DMOADs) which targeting osteoarthritis pathogenesis, such as cartilage destruction, subchondral bone remodeling, and synovial inflammation. These DMOADs are pursued not only to relief pain and improve joint function, but also inhibit the progression of structural disorder [5]. Even if no DMOADs have been licensed, several potential candidates are under development $[2,5]$.

Mesenchymal stem cells (MSCs) have been shown to possess broad immunoregulatory and anti-inflammatory abilities and are capable to suppress all immune cells that play an important pathogenic role in the development and progression of OA [6]. In addition, MSCs promote endogenous proliferation of chondrocyte and inhibit chondrocyte apoptosis or cartilage degeneration by paracrine signaling, achieving cartilage regeneration and cartilage protection $[7,8]$. These mechanism of actions of MSCs may not only provide the effective improvement of pain and symptom control, but also provide the potential disease-modifying effects on KOA.

Preclinical research has successfully shown the benefit of intra-articular MSC therapy for OA in pain relief and functional improvement [9-12]. Moreover, the initial clinical trials have an encouraging outcome on pain relief and functional improvement in symptomatic knee OA following MSC therapy [13-16]. While most studies have used autologous MSCs, allogeneic MSCs appear to have an acceptable safety profile which is similar to autologous cells. It is worth mentioning that allogeneic MSCs allow the manufacturing of large batches of off-the-shelf MSC products, which would enhance the consistency and decrease the costs of cell therapy, and solve the problem of limited proliferation capability of MSCs from elder donors. So far, the potential concern of using allogeneic adipose-derived stem cells (ADSCs) and the data of allogenic MSC for the KOA treatment is relatively limited as compared with the autologous MSCs [14, 17-22]. Therefore, we conducted a randomized Phase I/II clinical trial to assess the safety and therapeutic potential of allogeneic ADSCs in patients with KOA.

\section{Methods}

\section{Preparation of ELIXCYTE ${ }^{\circledR}$}

The donor who signed the informed consent for the preparation of ELIXCYTE was recruited under the supervision of an Institutional Review Board (CGMFIRB Number: 104-1790A3) and was screened and tested in compliance with eligibility determination guidance issued by Taiwan Food and Drug Administration. The adipose tissue was collected from eligible donor via ultrasonic-assisted liposuction and was transferred to UnicoCell BioMed Company under hypothermal condition within $6 \mathrm{~h}$. The stromal vascular fraction (SVF) was isolated by digesting the adipose tissue with type I collagenase, centrifuged, and cultured at $37^{\circ} \mathrm{C}$ in a humidified carbon dioxide incubator for primary culture. The adherent and highly proliferated cells termed ADSCs were detached and propagated to the passage 7 and within predefined population doubling level. The ADSCs were then harvested and formulated at a density of $8 \times 10^{6}$ cells $/ \mathrm{mL}$ with cryoprotectant CryoStor ${ }^{\circledR}$ CS10 (BioLife Solutions). Release testing of ELIXCYTE ${ }^{\circledR}$ for microbiological evaluation (including mycoplasma, sterility, and endotoxin tests) was employed to ensure safety. The product characteristics such as identity (including MSC markers, viable cell count, and cell viability), and tri-lineage differentiation properties were also assessed.

\section{Trial design}

This phase I/II study, named as adipose-derived stem cells (ADSCs) for knee osteoarthritis, was planned as a randomized, single-blind, active-control study to evaluate the safety and efficacy of allogeneic ADSCs (ELIXCYTE ${ }^{\circledR}$ ) 
intra-articular injection to patients with KOA. The study was registered on clinicaltrail.gov (NCT02784964) and was approved by Institutional Review Board to the tenets of the Declaration of Helsinki. There were two study sites in this trial, including Linkou Chang Gung Memorial Hospital and Taipei Veterans General Hospital in Taiwan. Permuted block randomization stratified by the center method was applied to generate randomization codes before study start, statistician from contract research organization (CRO) generated the random allocation sequence, investigators of this study enrolled participants, and the sponsor who assigned participants to interventions. Phase I of this study schemed to recruit 6 evaluable patients, and the permute block size was 6 . Eligible patients would be randomly assigned in 2:1 ratio to investigational product treatment group (ELIXCYTE ${ }^{\circledR}$ $64 \times 10^{6}$ cells, $64 \mathrm{M}$ ) or active-control group (Hya Joint Plus synovial fluid supplement $3 \mathrm{~mL}$, SciVision Biotech Inc).

Phase II of this study schemed to recruit 36 evaluable patients, and the permute block size was 9. Eligible patients would be randomly assigned in 2:3:3:1 ratio to investigational product treatment group (ELIXCYTE ${ }^{\circledR}$ $64 \times 10^{6}$ cells, $64 \mathrm{M}$; ELIXCYTE ${ }^{\circledR} 32 \times 10^{6}$ cells, $32 \mathrm{M}$; ELIXCYTE $^{\circledR} 16 \times 10^{6}$ cells, $16 \mathrm{M}$ ) or active-control group. Each eligible patient would have exactly one "target knee" for efficacy evaluation for this study. The target knee would be the knee meeting entry criteria and be administered on day 1 with the treatment upon the patient was randomly assigned. Subjects were followed for 96 weeks including scheduled visits at Weeks 2, 4, 12, 24 (main study), 36, 48 (extension study), 72, and 96 (structural study) after administration.

\section{Participants}

This study was planned to enroll about 56 subjects for 42 evaluable subjects. All subjects gave written informed consent to participate in the study prior to enrollment. Eligible participants included patients aged $40-80$ years (inclusive) with Kellgren-Lawrence (KL) grading I-III, as determined by American College of Rheumatology (ACR) criteria for KOA and WOMAC pain score 7-17. We excluded those with surgery history on the target knee joint, previous intra-articular intervention on the target knee joint within past 3 months, hypersensitivity to any component used in the study, inadequate hematologic and hepatic function, human immunodeficiency virus (HIV) infection or body mass index (BMI) greater than $35 \mathrm{~kg} / \mathrm{m}^{2}$. Patients already participated in any other interventional study within 4 weeks of entering the study were also excluded. Treatment applied to target knee area, or analgesics except for acetaminophen and NSAIDs would be prohibited from patients.

\section{Efficacy outcome measures}

The primary endpoint was the change in WOMAC pain score from baseline to week 24 after treatment. Secondary outcomes included change from baseline to posttreatment visit in WOMAC stiffness, physical function, and total score; VAS for pain and KSCRS score.

\section{Safety assessment}

The safety assessment was the incidence of AEs and SAEs.

\section{Clinical laboratory evaluation}

Laboratory assessments including hematology and biochemistry were performed at the Screening, Week 24, $36,48,72$, and 96 visits. The measured items were listed as below: for hematology: white blood cells (WBC), neutrophils, lymphocytes, monocytes, eosinophils, basophils, hemoglobin, hematocrit, platelet, and red blood cells (RBC); for biochemistry: aspartate aminotransferase, alanine aminotransferase, serum creatinine, blood urea nitrogen (BUN), and albumin; for immunogenicity, tumor necrosis factor alpha (TNF- $\alpha$ ), CD4, and CD8 were measured at screening, Week 4, and 24 visits.

\section{Statistical analysis}

All phase I and phase II data were pooled for statistical analysis. Efficacy endpoints and safety endpoints were analyzed on the intent-to-treat (ITT) population. Demographics and baseline characteristics were analyzed by using a two-sample t-test, Wilcoxon rank-sum test or Fisher's exact test as appropriate to ensure comparability between treatments. A two-sample t-test was applied for $p$-value (pairwise) in the statistic table, Wilcoxon ranksum test was applied if the normality assumption was not valid ( $p$-value of Shapiro-Wilk normality test $\leq 0.05$ ). The efficacy endpoints, changes in WOMAC, VAS and KSCRS were analyzed by using ANCOVA incorporating treatment effect and baseline as covariate or by Wilcoxon rank-sum test. For safety analyses, the incidence of adverse events was tabulated by treatment groups and by physiological systems as appropriate.

Pairwise treatment group comparisons were conducted with a significance level of 0.05 without alpha adjustment. For efficacy endpoints, a 95\% two-sided confidence interval on the difference of each treatment was provided as appropriate.

\section{Results}

Patient disposition and baseline characteristics

Total 64 subjects were screened, where 7 were screen failures, resulted in 57 (89\%) subjects being eligible and 
randomized. Among them, 8 (14\%) subjects received HA and 17 (29.8\%) subjects received ELIXCYTE ${ }^{\circledR} 16 \mathrm{M}$, 17 (29.8\%) subjects received ELIXCYTE ${ }^{\circledR} 32 \mathrm{M}$, and 15 (26.3\%) subjects received ELIXCYTE ${ }^{\circledR} 64 \mathrm{M}$ within the study period (Fig. 1). The ITT population consisted of the 57 randomized patients who received at least one dose of single-blind treatment.

Table 1 shows the demographic and baseline characteristics of the ITT patient population. Among 57 enrolled study subjects, 11 (19.3\%) were male and 46 (80.7\%) were female. The 57 eligible subjects were $($ mean \pm SD) $67.6 \pm 6.60$ years of age, $155.2 \pm 7.92 \mathrm{~cm}$ in height, $63.9 \pm 9.64 \mathrm{~kg}$ in weight, and $26.54 \pm 3.673 \mathrm{~kg} / \mathrm{m}^{2}$ in BMI. The baseline $\mathrm{KL}$ grade of the target knees was either grade II $(37 / 57,64.9 \%)$ or grade III $(20 / 57,35.1 \%)$. The average duration of KOA since diagnosed was 2.96 years. No particular differences between individual ELIXCYTE $^{\circledR}$ dose and HA groups were observed in demographic data and KOA history.

\section{Safety}

The summary of all treatment-emergent adverse events (TEAEs) during Week 0-24 and Week 24-96 is listed in Table 2, and all the treatment-related AEs were observed during week 0-24. Forty-four subjects (77.2\%) reported at least one TEAE and 25 subjects (43.9\%) experiencing at least one treatment-related AEs during Week 0-24. There were 3 subjects (5.3\%) reported AEs with grade $\geq$ 3 , including 2 subjects (11.8\%) in middle-dose group and 1 subject (6.7\%) in high-dose group, which was judged as treatment-related AE. During Week 24-96, 31 subjects (54.4\%) reported at least one TEAEs. There were 6 SAEs recorded during the whole study period (Week 0-96), and none of them were judged as treatment-related SAE. Moreover, no SUSAR or death was reported in this study.

Table 3 shows the TEAEs during Week $0-24$ by system organ class (SOC). The most frequent TEAEs during Week 0-24 in total population were "Musculoskeletal and connective tissue disorders" (40.4\%), followed by "General disorders and administration site conditions" (29.8\%), "Infections and infestation" (21.1\%), and "Gastrointestinal disorders" (12.3\%). The frequency of other TEAEs was lower than $10.0 \%$. TEAEs observed during Week 24-96 are presented in Additional file 2: Table S1.

As for treatment-related AEs, the most frequent AEs were "Injection site joint pain" (15.8\%), followed by "Arthralgia" (14.0\%), "Injection site joint swelling" (12.3\%), and "Joint swelling" (10.5\%). The frequency of other treatment-related AEs was lower than 5\%. There was no treatment-related $\mathrm{AE}$ reported during Week 24-96. The summary of treatment-related AEs is presented in Table 4.

Additional file 1 showed that there was no clinically significant (NCS) abnormality in hematology observed in the subjects during the study, and most biochemistry values were normal or abnormal but NCS. However, the abnormal and clinically significant laboratory values of aspartate aminotransferase (AST) were observed in $1(7.7 \%)$ subject in $32 \mathrm{M}$ and $1(8.3 \%)$ subject in the

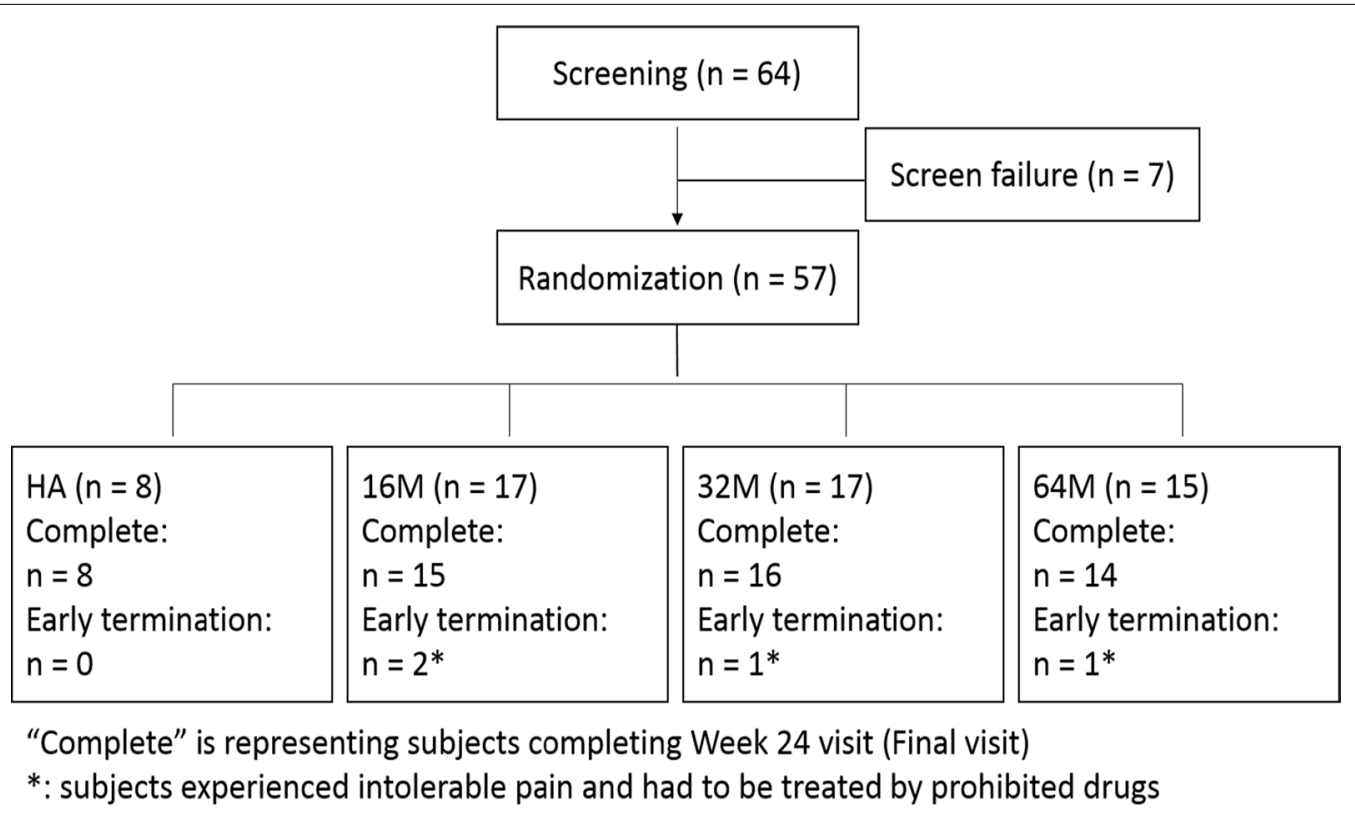

Fig. 1 Patient flow diagram showing subject numbers for screening, randomization, and treatment assignment 
Table 1 Summary of demographic data_ITT population at the screening visit

\begin{tabular}{|c|c|c|c|c|c|}
\hline \multirow[t]{2}{*}{ Demographic data } & \multicolumn{5}{|c|}{ ITT population } \\
\hline & $\mathrm{HA}$ & $16 \mathrm{M}$ & $32 \mathrm{M}$ & $64 M$ & Total \\
\hline N (Missing) & $8(0)$ & $17(0)$ & $17(0)$ & $15(0)$ & $57(0)$ \\
\hline \multicolumn{6}{|l|}{ Age (year) } \\
\hline Mean (SD) & $70.5(8.37)$ & $67.7(6.84)$ & $68.6(6.45)$ & $64.9(4.91)$ & $67.6(6.60)$ \\
\hline Median (IQR) & $73.5(13.0)$ & $66.0(5.0)$ & $68.0(9.0)$ & $65.0(8.0)$ & $67.0(10.0)$ \\
\hline Q1 Q3 & $64.0 \sim 77.0$ & $65.0 \sim 70.0$ & $65.0 \sim 74.0$ & $60.0 \sim 68.0$ & $63.0 \sim 73.0$ \\
\hline Min Max & $56.0 \sim 79.0$ & $51.0 \sim 78.0$ & $55.0 \sim 78.0$ & $59.0 \sim 75.0$ & $51.0 \sim 79.0$ \\
\hline$p$-value (T test) & - & 0.3835 & 0.5356 & 0.0531 & - \\
\hline \multicolumn{6}{|l|}{ Gender } \\
\hline Male & $3(37.5 \%)$ & $3(17.6 \%)$ & $2(11.8 \%)$ & $3(20.0 \%)$ & $11(19.3 \%)$ \\
\hline Female & $5(62.5 \%)$ & $14(82.4 \%)$ & 15 (88.2\%) & $12(80.0 \%)$ & $46(80.7 \%)$ \\
\hline$p$-value (FISHER) & - & 0.3442 & 0.2833 & 0.6214 & - \\
\hline \multicolumn{6}{|l|}{ Body height (cm) } \\
\hline Mean (SD) & $154.0(12.42)$ & $154.7(7.51)$ & $155.0(6.75)$ & $156.5(7.36)$ & $155.2(7.92)$ \\
\hline Median (IQR) & $148.8(23.9)$ & $155.0(7.5)$ & $153.5(6.9)$ & $155.4(8.5)$ & $154.2(9.1)$ \\
\hline Q1 Q3 & $143.8 \sim 167.7$ & $149.5 \sim 157.0$ & $151.0 \sim 157.9$ & $152.0 \sim 160.5$ & $149.5 \sim 158.6$ \\
\hline Min Max & $141.0 \sim 170.3$ & $142.0 \sim 177.2$ & $147.7 \sim 176.0$ & $145.3 \sim 168.3$ & $141.0 \sim 177.2$ \\
\hline$p$-value (Wilcox_t) & - & 0.5275 & 0.3907 & 0.4656 & - \\
\hline \multicolumn{6}{|l|}{ Body weight (Kg) } \\
\hline Mean (SD) & $60.5(10.80)$ & $66.0(6.80)$ & $64.2(10.97)$ & $62.9(10.47)$ & $63.9(9.64)$ \\
\hline Median (IQR) & $64.3(18.5)$ & $65.0(6.9)$ & $63.2(13.1)$ & $63.8(16.6)$ & $63.8(12.9)$ \\
\hline Q1 Q3 & $49.4 \sim 67.8$ & $62.0 \sim 68.9$ & $56.4 \sim 69.5$ & $55.7 \sim 72.3$ & $56.6 \sim 69.5$ \\
\hline Min Max & $46.2 \sim 74.6$ & $55.6 \sim 77.4$ & $48.0 \sim 87.2$ & $42.3 \sim 80.2$ & $42.3 \sim 87.2$ \\
\hline$p$-value (T test) & - & 0.1321 & 0.4293 & 0.6015 & - \\
\hline \multicolumn{6}{|l|}{$B M I\left(K g / m^{2}\right)$} \\
\hline Mean (SD) & $25.47(3.494)$ & $27.65(3.026)$ & $26.72(4.192)$ & $25.66(3.782)$ & $26.54(3.673)$ \\
\hline Median (IQR) & $24.64(3.89)$ & $28.28(4.68)$ & $25.98(3.68)$ & $25.67(5.78)$ & $25.98(5.22)$ \\
\hline Q1 Q3 & $23.33 \sim 27.21$ & $25.03 \sim 29.70$ & $24.56 \sim 28.24$ & $22.90 \sim 28.68$ & $23.83 \sim 29.05$ \\
\hline Min Max & $21.09 \sim 32.29$ & $23.14 \sim 32.26$ & $20.37 \sim 34.59$ & $19.39 \sim 31.33$ & $19.39 \sim 34.59$ \\
\hline$p$-value (T test) & - & 0.1232 & 0.4724 & 0.9088 & - \\
\hline \multicolumn{6}{|c|}{$\begin{array}{l}\text { Kellgren-Lawrence grade on target } \\
\text { knee }\end{array}$} \\
\hline Grade II & $5(62.5 \%)$ & $10(58.8 \%)$ & $10(58.8 \%)$ & $12(80.0 \%)$ & 37 (64.9\%) \\
\hline Grade III & $3(37.5 \%)$ & $7(41.2 \%)$ & $7(41.2 \%)$ & $3(20.0 \%)$ & $20(35.1 \%)$ \\
\hline$p$-value (FISHER) & - & 1.0000 & 1.0000 & 0.6214 & - \\
\hline \multicolumn{6}{|l|}{ Disease duration (years) } \\
\hline Mean (SD) & $2.44(2.367)$ & $2.97(1.755)$ & $3.68(7.198)$ & $2.39(1.634)$ & $2.96(4.164)$ \\
\hline Median (IQR) & $2.05(2.24)$ & $2.97(2.13)$ & $2.04(2.48)$ & $2.08(2.48)$ & $2.26(2.24)$ \\
\hline Q1 Q3 & $0.81 \sim 3.05$ & $2.06 \sim 4.20$ & $0.20 \sim 2.68$ & $1.12 \sim 3.59$ & $1.12 \sim 3.36$ \\
\hline Min Max & $0.10 \sim 7.58$ & $0.00 \sim 5.87$ & $0.00 \sim 29.68$ & $0.16 \sim 5.86$ & $0.00 \sim 29.68$ \\
\hline$p$-value (Wilcox_t) & - & 0.3755 & 0.7514 & 0.8733 & - \\
\hline
\end{tabular}

For $\mathrm{Cl}$ and $p$-value (between groups), FISHER denotes Fisher's exact test, T denotes two-sample t-test, Wilcox_t denotes Wilcoxon rank-sum test (Mann-Whitney U Test) in $t$ approximation

$64 \mathrm{M}$ at Week 48 which were not treatment related. The CS values of ALT were also observed in 1 (7.7\%) subject in the $32 \mathrm{M}$ and 1 (8.3\%) subject in the $64 \mathrm{M}$ at Week 48. Regarding immunogenicity, most biochemistry values were normal or abnormal but NCS, except 1 (5.9\%) abnormal and clinically significant TNF- $\alpha$ result was found in the 32M group at Week 4 . These abnormal values were mainly related to medical history or adverse events of the subjects (Additional file 1, Table 18). 
Table 2 Summary of treatment-emergent adverse events (TEAEs)

\begin{tabular}{|c|c|c|c|c|c|c|c|c|c|c|}
\hline \multirow{2}{*}{$\begin{array}{l}\text { Treatment-emergent adverse events } \\
\text { [Event \# (E)/subject \# (S) (\%)] }\end{array}$} & \multicolumn{2}{|c|}{$\begin{array}{l}\mathrm{HA} \\
\mathrm{N}=8\end{array}$} & \multicolumn{2}{|c|}{$\begin{array}{l}16 M \\
N=17\end{array}$} & \multicolumn{2}{|c|}{$\begin{array}{l}32 M \\
N=17\end{array}$} & \multicolumn{2}{|c|}{$\begin{array}{l}64 M \\
N=15\end{array}$} & \multicolumn{2}{|c|}{$\begin{array}{l}\text { Total } \\
N=57\end{array}$} \\
\hline & $\mathrm{E}$ & $\mathrm{S}(\%)$ & $\mathrm{E}$ & $\mathrm{S}(\%)$ & $\mathrm{E}$ & $\mathrm{S}(\%)$ & $\mathrm{E}$ & $\mathrm{S}(\%)$ & $\mathrm{E}$ & $\mathrm{S}(\%)$ \\
\hline \multicolumn{11}{|l|}{ Week 0-24 (early TEAEs) } \\
\hline Subjects with AEs & 11 & $4(50.0 \%)$ & 40 & $15(88.2 \%)$ & 41 & $14(82.4 \%)$ & 25 & $11(73.3 \%)$ & 117 & $44(77.2 \%)$ \\
\hline Subjects with treatment-related AEs & 1 & $1(12.5 \%)$ & 12 & $6(35.3 \%)$ & 16 & $10(58.8 \%)$ & 17 & $8(53.3 \%)$ & 46 & $25(43.9 \%)$ \\
\hline Subjects with grade $\geq 3 \mathrm{AEs}$ & 0 & $0(0.0 \%)$ & 0 & $0(0.0 \%)$ & 2 & $2(11.8 \%)$ & 1 & $1(6.7 \%)$ & 3 & $3(5.3 \%)$ \\
\hline Subjects with grade $\geq 3$ treatment-related AEs & 0 & $0(0.0 \%)$ & 0 & $0(0.0 \%)$ & 0 & $0(0.0 \%)$ & 1 & $1(6.7 \%)$ & 1 & $1(1.8 \%)$ \\
\hline Subjects with treatment-modified AE & 0 & $0(0.0 \%)$ & 0 & $0(0.0 \%)$ & 0 & $0(0.0 \%)$ & 0 & $0(0.0 \%)$ & 0 & $0(0.0 \%)$ \\
\hline Subjects with SAEs & 0 & $0(0.0 \%)$ & 0 & $0(0.0 \%)$ & 1 & $1(5.9 \%)$ & 0 & $0(0.0 \%)$ & 1 & $1(1.8 \%)$ \\
\hline Subjects with SUSARs & 0 & $0(0.0 \%)$ & 0 & $0(0.0 \%)$ & 0 & $0(0.0 \%)$ & 0 & $0(0.0 \%)$ & 0 & $0(0.0 \%)$ \\
\hline \multicolumn{11}{|l|}{ Week 24-96 (Long-term TEAEs) } \\
\hline Subjects with AEs & 16 & $7(87.5 \%)$ & 19 & $7(41.2 \%)$ & 23 & $8(47.1 \%)$ & 25 & $9(60.0 \%)$ & 83 & $31(54.4 \%)$ \\
\hline Subjects with SAEs & 0 & $0(0.0 \%)$ & 0 & $0(0.0 \%)$ & 3 & $2(11.8 \%)$ & 2 & $2(13.3 \%)$ & 5 & $4(7.0 \%)$ \\
\hline Subjects with SUSARs & 0 & $0(0.0 \%)$ & 0 & $0(0.0 \%)$ & 0 & $0(0.0 \%)$ & 0 & $0(0.0 \%)$ & 0 & $0(0.0 \%)$ \\
\hline
\end{tabular}

Treatment-related TEAEs included possible, probable, and definite in relation to study treatment

$A E$, adverse event; TEAE, treatment emergent adverse event; $S A E$, serious adverse event; SUSAR, suspected unexpected serious adverse reaction

Table 3 Summary of adverse events during week 0-24 (early TEAEs)

\begin{tabular}{|c|c|c|c|c|c|c|c|c|c|c|}
\hline \multirow{2}{*}{$\begin{array}{l}\text { Adverse event (Week 0-24) } \\
\text { [Event \# (E)/Subject \# (S) (\%)] }\end{array}$} & \multicolumn{2}{|c|}{$\begin{array}{l}\mathrm{HA} \\
N=8\end{array}$} & \multicolumn{2}{|c|}{$\begin{array}{l}16 \mathrm{M} \\
N=17\end{array}$} & \multicolumn{2}{|c|}{$\begin{array}{l}32 \mathrm{M} \\
N=17\end{array}$} & \multicolumn{2}{|c|}{$\begin{array}{l}64 \mathrm{M} \\
N=15\end{array}$} & \multicolumn{2}{|c|}{$\begin{array}{l}\text { Total } \\
N=57\end{array}$} \\
\hline & $E$ & $\mathrm{~S}(\%)$ & $\mathrm{E}$ & $\mathrm{S}(\%)$ & $\mathrm{E}$ & $\mathrm{S}(\%)$ & $\mathrm{E}$ & $\mathrm{S}(\%)$ & $\mathrm{E}$ & $\mathrm{S}(\%)$ \\
\hline At least one below & 11 & $4(50.0 \%)$ & 40 & 15 (88.2\%) & 41 & $14(82.4 \%)$ & 25 & $11(73.3 \%)$ & 117 & $44(77.2 \%)$ \\
\hline Cardiac disorders & 1 & $1(12.5 \%)$ & 0 & $0(0.0 \%)$ & 1 & $1(5.9 \%)$ & 0 & $0(0.0 \%)$ & 2 & $2(3.5 \%)$ \\
\hline Eye disorders & 1 & $1(12.5 \%)$ & 2 & $2(11.8 \%)$ & 0 & $0(0.0 \%)$ & 0 & $0(0.0 \%)$ & 3 & $3(5.3 \%)$ \\
\hline Gastrointestinal disorders & 1 & $1(12.5 \%)$ & 3 & $2(11.8 \%)$ & 4 & $3(17.6 \%)$ & 1 & $1(6.7 \%)$ & 9 & $7(12.3 \%)$ \\
\hline General disorders and administration site conditions & 1 & $1(12.5 \%)$ & 4 & $3(17.6 \%)$ & 7 & $7(41.2 \%)$ & 7 & $6(40.0 \%)$ & 19 & $17(29.8 \%)$ \\
\hline Hepatobiliary disorders & 0 & $0(0.0 \%)$ & 0 & $0(0.0 \%)$ & 1 & $1(5.9 \%)$ & 0 & $0(0.0 \%)$ & 1 & $1(1.8 \%)$ \\
\hline Infections and infestations & 1 & $1(12.5 \%)$ & 6 & $6(35.3 \%)$ & 3 & $2(11.8 \%)$ & 4 & $3(20.0 \%)$ & 14 & $12(21.1 \%)$ \\
\hline Injury, poisoning, and procedural complications & 0 & $0(0.0 \%)$ & 2 & $1(5.9 \%)$ & 1 & $1(5.9 \%)$ & 0 & $0(0.0 \%)$ & 3 & $2(3.5 \%)$ \\
\hline Investigations & 0 & $0(0.0 \%)$ & 0 & $0(0.0 \%)$ & 1 & $1(5.9 \%)$ & 0 & $0(0.0 \%)$ & 1 & $1(1.8 \%)$ \\
\hline Metabolism and nutrition disorders & 0 & $0(0.0 \%)$ & 2 & $1(5.9 \%)$ & 0 & $0(0.0 \%)$ & 0 & $0(0.0 \%)$ & 2 & $1(1.8 \%)$ \\
\hline Musculoskeletal and connective tissue disorders & 5 & $2(25.0 \%)$ & 15 & $6(35.3 \%)$ & 13 & $10(58.8 \%)$ & 11 & $5(33.3 \%)$ & 44 & $23(40.4 \%)$ \\
\hline $\begin{array}{l}\text { Neoplasms benign, malignant, and unspecified } \\
\text { (including cysts and polyps) }\end{array}$ & 0 & $0(0.0 \%)$ & 2 & $2(11.8 \%)$ & 1 & $1(5.9 \%)$ & 0 & $0(0.0 \%)$ & 3 & $3(5.3 \%)$ \\
\hline Nervous system disorders & 1 & $1(12.5 \%)$ & 1 & $1(5.9 \%)$ & 2 & $2(11.8 \%)$ & 1 & $1(6.7 \%)$ & 5 & $5(8.8 \%)$ \\
\hline Psychiatric disorders & 0 & $0(0.0 \%)$ & 0 & $0(0.0 \%)$ & 1 & $1(5.9 \%)$ & 0 & $0(0.0 \%)$ & 1 & $1(1.8 \%)$ \\
\hline Renal and urinary disorders & 0 & $0(0.0 \%)$ & 0 & $0(0.0 \%)$ & 1 & $1(5.9 \%)$ & 0 & $0(0.0 \%)$ & 1 & $1(1.8 \%)$ \\
\hline Respiratory, thoracic, and mediastinal disorders & 0 & $0(0.0 \%)$ & 1 & $1(5.9 \%)$ & 2 & $2(11.8 \%)$ & 1 & $1(6.7 \%)$ & 4 & $4(7.0 \%)$ \\
\hline Skin and subcutaneous tissue disorders & 0 & $0(0.0 \%)$ & 1 & $1(5.9 \%)$ & 1 & $1(5.9 \%)$ & 0 & $0(0.0 \%)$ & 2 & $2(3.5 \%)$ \\
\hline Surgical and medical procedures & 0 & $0(0.0 \%)$ & 1 & $1(5.9 \%)$ & 2 & $1(5.9 \%)$ & 0 & $0(0.0 \%)$ & 3 & $2(3.5 \%)$ \\
\hline
\end{tabular}

Dictionary: MedDRA version 19.0

\section{Efficacy: primary outcomes}

The WOMAC pain score at week 24 was used to evaluate the primary endpoint. Under no significant difference between baseline values of HA and all ELIXCYTE ${ }^{\circledR}$ groups, all groups had significant reductions of pain score from baseline to post-treatment through Week 4 to 48 (Additional file 2: Table S2 for detail $p$-value information). In addition, the earlier significant reductions of pain score at Week 2 were observed in $16 \mathrm{M}, 32 \mathrm{M}$, and Pooled groups compared to those in HA group (16 M: 
Table 4 Summary of treatment-related adverse events during week 0-24

\begin{tabular}{|c|c|c|c|c|c|c|c|c|c|c|}
\hline \multirow{2}{*}{$\begin{array}{l}\text { Treatment-relative adverse event (week 0-24) } \\
\text { [Event \# (E)/subject \# (S) (\%)] }\end{array}$} & \multicolumn{2}{|c|}{$\begin{array}{l}\mathrm{HA} \\
N=8\end{array}$} & \multicolumn{2}{|c|}{$\begin{array}{l}16 \mathrm{M} \\
N=17\end{array}$} & \multicolumn{2}{|c|}{$\begin{array}{l}32 \mathrm{M} \\
N=17\end{array}$} & \multicolumn{2}{|c|}{$\begin{array}{l}64 \mathrm{M} \\
N=15\end{array}$} & \multicolumn{2}{|c|}{$\begin{array}{l}\text { Total } \\
N=57\end{array}$} \\
\hline & $E$ & $\mathrm{~S}(\%)$ & $\mathrm{E}$ & $\mathrm{S}(\%)$ & $\mathrm{E}$ & $\mathrm{S}(\%)$ & $\mathrm{E}$ & $\mathrm{S}(\%)$ & $\mathrm{E}$ & $\mathrm{S}(\%)$ \\
\hline At least one below & 1 & $1(12.5 \%)$ & 12 & $6(35.3 \%)$ & 16 & $10(58.8 \%)$ & 17 & $8(53.3 \%)$ & 46 & $25(43.9 \%)$ \\
\hline General disorders and administration site conditions & 1 & $1(12.5 \%)$ & 4 & $3(17.6 \%)$ & 7 & $7(41.2 \%)$ & 7 & $6(40.0 \%)$ & 19 & $17(29.8 \%)$ \\
\hline Injection site erythema & 0 & $0(0.0 \%)$ & 0 & $0(0.0 \%)$ & 1 & $1(5.9 \%)$ & 0 & $0(0.0 \%)$ & 1 & $1(1.8 \%)$ \\
\hline Injection site joint effusion & 0 & $0(0.0 \%)$ & 0 & $0(0.0 \%)$ & 0 & $0(0.0 \%)$ & 1 & $1(6.7 \%)$ & 1 & $1(1.8 \%)$ \\
\hline Injection site joint inflammation & 0 & $0(0.0 \%)$ & 1 & $1(5.9 \%)$ & 0 & $0(0.0 \%)$ & 0 & $0(0.0 \%)$ & 1 & $1(1.8 \%)$ \\
\hline Injection site joint pain & 1 & $1(12.5 \%)$ & 1 & $1(5.9 \%)$ & 3 & $3(17.6 \%)$ & 4 & $4(26.7 \%)$ & 9 & $9(15.8 \%)$ \\
\hline Injection site joint swelling & 0 & $0(0.0 \%)$ & 2 & $2(11.8 \%)$ & 3 & $3(17.6 \%)$ & 2 & $2(13.3 \%)$ & 7 & $7(12.3 \%)$ \\
\hline Infections and infestations & 0 & $0(0.0 \%)$ & 1 & $1(5.9 \%)$ & 0 & $0(0.0 \%)$ & 0 & $0(0.0 \%)$ & 1 & $1(1.8 \%)$ \\
\hline$\sim$ Pharyngitis & 0 & $0(0.0 \%)$ & 1 & $1(5.9 \%)$ & 0 & $0(0.0 \%)$ & 0 & $0(0.0 \%)$ & 1 & $1(1.8 \%)$ \\
\hline Injury, poisoning, and procedural complications & 0 & $0(0.0 \%)$ & 0 & $0(0.0 \%)$ & 1 & $1(5.9 \%)$ & 0 & $0(0.0 \%)$ & 1 & $1(1.8 \%)$ \\
\hline$\sim$ Procedural vomiting & 0 & $0(0.0 \%)$ & 0 & $0(0.0 \%)$ & 1 & $1(5.9 \%)$ & 0 & $0(0.0 \%)$ & 1 & $1(1.8 \%)$ \\
\hline Investigations & 0 & $0(0.0 \%)$ & 0 & $0(0.0 \%)$ & 1 & $1(5.9 \%)$ & 0 & $0(0.0 \%)$ & 1 & $1(1.8 \%)$ \\
\hline$\sim$ Alpha tumor necrosis factor increased & 0 & $0(0.0 \%)$ & 0 & $0(0.0 \%)$ & 1 & $1(5.9 \%)$ & 0 & $0(0.0 \%)$ & 1 & $1(1.8 \%)$ \\
\hline Musculoskeletal and connective tissue disorders & 0 & $0(0.0 \%)$ & 4 & $2(11.8 \%)$ & 6 & $5(29.4 \%)$ & 10 & $4(26.7 \%)$ & 20 & $11(19.3 \%)$ \\
\hline$\sim$ Arthralgia & 0 & $0(0.0 \%)$ & 3 & $2(11.8 \%)$ & 2 & $2(11.8 \%)$ & 6 & $4(26.7 \%)$ & 11 & $8(14.0 \%)$ \\
\hline$\sim$ Joint stiffness & 0 & $0(0.0 \%)$ & 0 & $0(0.0 \%)$ & 1 & $1(5.9 \%)$ & 2 & $1(6.7 \%)$ & 3 & $2(3.5 \%)$ \\
\hline Joint swelling & 0 & $0(0.0 \%)$ & 1 & $1(5.9 \%)$ & 3 & $3(17.6 \%)$ & 2 & $2(13.3 \%)$ & 6 & $6(10.5 \%)$ \\
\hline $\begin{array}{l}\text { Neoplasms benign, malignant, and unspecified } \\
\text { (including cysts and polyps) }\end{array}$ & 0 & $0(0.0 \%)$ & 1 & $1(5.9 \%)$ & 0 & $0(0.0 \%)$ & 0 & $0(0.0 \%)$ & 1 & $1(1.8 \%)$ \\
\hline Pharyngeal neoplasm benign & 0 & $0(0.0 \%)$ & 1 & $1(5.9 \%)$ & 0 & $0(0.0 \%)$ & 0 & $0(0.0 \%)$ & 1 & $1(1.8 \%)$ \\
\hline Nervous system disorders & 0 & $0(0.0 \%)$ & 1 & $1(5.9 \%)$ & 1 & $1(5.9 \%)$ & 0 & $0(0.0 \%)$ & 2 & $2(3.5 \%)$ \\
\hline$\sim$ Dizziness & 0 & $0(0.0 \%)$ & 1 & $1(5.9 \%)$ & 1 & $1(5.9 \%)$ & 0 & $0(0.0 \%)$ & 2 & $2(3.5 \%)$ \\
\hline Skin and subcutaneous tissue disorders & 0 & $0(0.0 \%)$ & 1 & $1(5.9 \%)$ & 0 & $0(0.0 \%)$ & 0 & $0(0.0 \%)$ & 1 & $1(1.8 \%)$ \\
\hline$\sim$ Cold sweat & 0 & $0(0.0 \%)$ & 1 & $1(5.9 \%)$ & 0 & $0(0.0 \%)$ & 0 & $0(0.0 \%)$ & 1 & $1(1.8 \%)$ \\
\hline
\end{tabular}

Dictionary: MedDRA version 19.0

$P=0.0024 ; 32$ M: $P=0.0040$; Pooled $P<0.0001)$. The significant difference in mean WOMAC pain scores was observed at Week 4 in $64 \mathrm{M}$ and Pooled groups in comparison with the HA group (64 M: $P=0.0026$; Pooled: $P=0.0381$ ) (Fig. 2a, Additional file 2: Table S2).

\section{Efficacy: secondary outcomes}

The baseline values of HA and all ELIXCYTE ${ }^{\circledR}$ groups were similar in WOMAC score (total, stiffness, and functional limitation), VAS score for pain, and KSCRS score (objective knee indicators, symptoms, and functional activities), except KSCRS score-patient satisfaction for the $16 \mathrm{M}(P=0.0253)$. (Individual $p$ values are shown in supplement tables.)

All ELIXCYTE ${ }^{\circledR}$ groups had a significant reduction in WOMAC total score, stiffness, and functional limitation score from baseline to post-treatment at Week 12 to 48 (Additional file 2: Table S3, S4, and S5 for detailed $p$-value information). The earlier significant decreases of WOMAC total score from baseline to post-treatment were observed at Week 2 in $16 \mathrm{M}, 32 \mathrm{M}$, and Pooled groups (16 M: $P=0.0129$; $32 \mathrm{M}: P=0.0088$; Pooled $P<0.0001$ ), and at Week 4 in $16 \mathrm{M}, 32 \mathrm{M}, 64 \mathrm{M}$, and Pooled groups (16 M: $P=0.0004 ; 32 \mathrm{M}: P<0.0001 ; 64 \mathrm{M}$ : $P=0.0001$; Pooled $P<0.0001)$. A significant difference in mean WOMAC total scores was observed at Week 4 in $16 \mathrm{M}, 32 \mathrm{M}, 64 \mathrm{M}$, Pooled groups (16 M: $P=0.0481$; 32 M: $P=0.0390$; 64 M: $P=0.0014$; Pooled $P=0.0075$ ), and Week 48 in $64 \mathrm{M}$ group in comparison with HA group $(P=0.0113)$ (Fig. 2b, Additional file 2: Table S3). A significant difference of mean WOMAC stiffness scores was observed at Week 4 in $16 \mathrm{M}, 32 \mathrm{M}, 64 \mathrm{M}$, and Pooled groups (16 M: $P=0.0369 ; 32 \mathrm{M}: P=0.0360 ; 64 \mathrm{M}$ : $P=0.0027$; Pooled $P=0.0073$ ), and at Week 48 in $64 \mathrm{M}$ in comparison with the HA group $(P=0.0402)$ (Fig. $2 \mathrm{C}$, Additional file 2: Table S4). In WOMAC functional limitation score, the earlier improvement from baseline to post-treatment was found at Week 2 in $16 \mathrm{M}, 32 \mathrm{M}$, and Pooled groups (16 M: $P=0.0173 ; 32 \mathrm{M}: P=0.0155$; Pooled $P<0.0001$ ), and at Week 4 in $16 \mathrm{M}, 32 \mathrm{M}, 64 \mathrm{M}$, and Pooled groups (16 M: $P=0.0010 ; 32 \mathrm{M}: P<0.0001$; 64 M: $P=0.0003$; Pooled $P<0.0001)$. In the comparison 

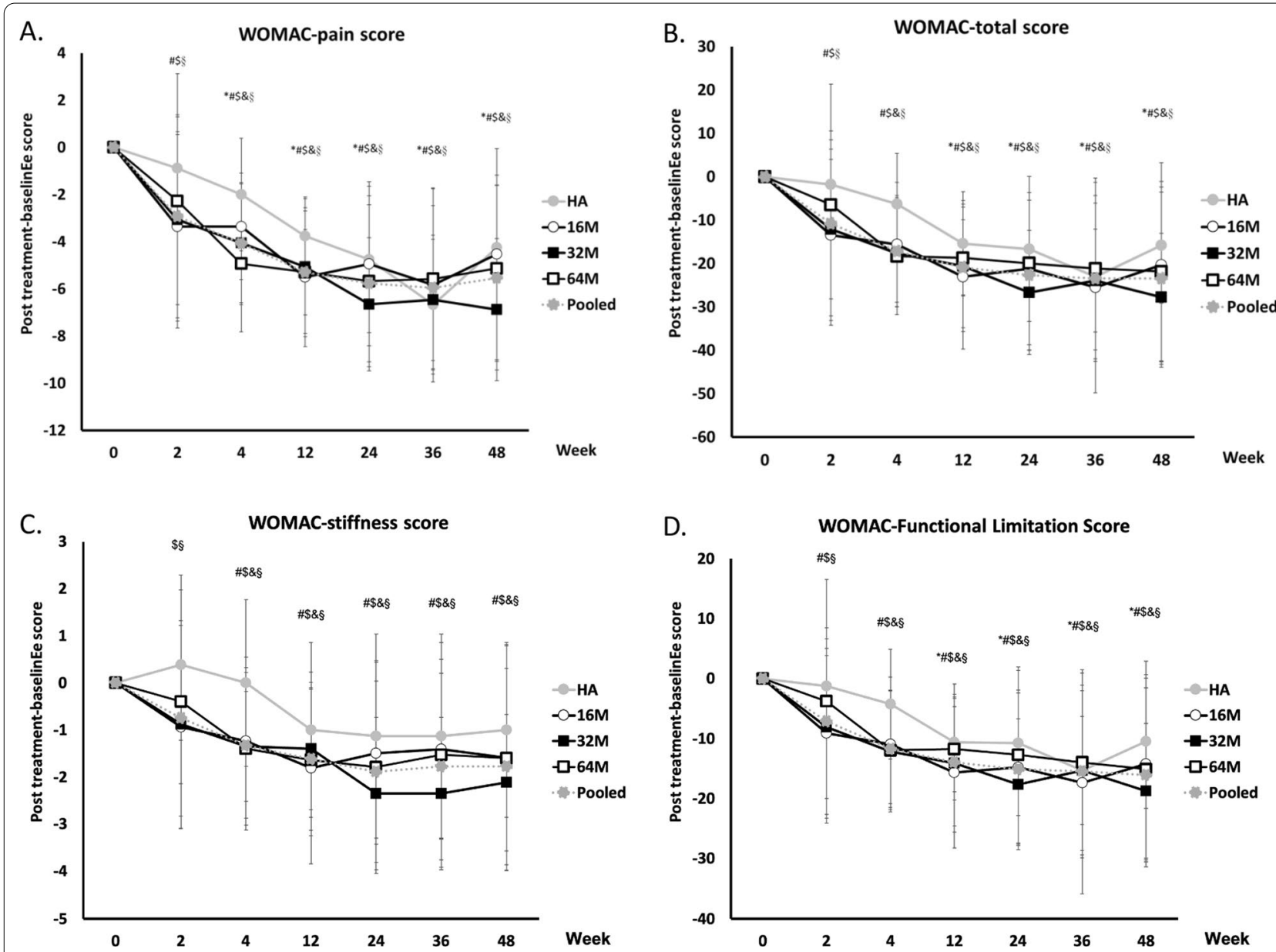

Fig. 2 Changes in WOMAC after intra-articular injection of ELIXCYTE ${ }^{\circledR}$ or HA at each time point. WOMAC pain (a), total (b), stiffness (c), and function limitation (d) scores are presented as mean \pm SD. 16 M, 32 M, 64 M, and HA=16, 32, 64 million of ELIXCYTE ${ }^{\circledR}$, and hyaluronic acid, respectively. The signs of significance, ${ }^{*}, \#, \$, \&, \S$ representing a $p$-value $<0.05$ between baseline and posttreatment score comparison for HA, $16 \mathrm{M}, 32 \mathrm{M}, 64 \mathrm{M}$, and Pooled group

of HA and 64 M group at Week 48, a significant difference in the mean of functional limitation scores of WOMAC was observed $(P=0.0070)$ (Fig. 2d, Additional file 2: Table S5).

The time to observe significant reductions of VAS pain scores from baseline to post-treatment for HA and individual ELIXCYTE ${ }^{\circledR}$ groups was Week 12 to 36, and Week 4 to 48 (Additional file 2: Table S6 for detail $p$-value information), respectively. Moreover, the earlier pain improvements were found at Week 2 in $16 \mathrm{M}, 64 \mathrm{M}$, and Pooled groups (16 M: $P=0.0121 ; 64 \mathrm{M}: P=0.0447$; Pooled: $P=0.0002)$. A significant difference in mean VAS scores was observed at Week 4 in $64 \mathrm{M}$ and Pooled groups (64 M: $P=0.0021$; Pooled: $P=0.0127$ ), at Week 12 in $16 \mathrm{M}, 32 \mathrm{M}, 64 \mathrm{M}$, and Pooled groups (16 M: $P=0.0026$; $32 \mathrm{M}: P=0.0439$; $64 \mathrm{M}: P=0.0003$; Pooled: $P=0.0014)$ in comparison with the HA group (Fig. 3, Additional file 2: Table S6).
There were significant increases of KSCRS objective knee indicator scores from baseline to post-treatment in $16 \mathrm{M}$ and Pooled groups at Weeks 12 to 48 (16 M: $P=0.0078,0.0264,0.0234$; Pooled: $P=0.0021$, $0.0105,0.0002)$ compared to a decrease of score in HA group. A significant increase of mean KSCRS scores was observed at Week 12 in $16 \mathrm{M}, 64 \mathrm{M}$, and Pooled groups (16 M: $P=0.0113$; $64 \mathrm{M}: P=0.0370$; Pooled: $P=0.0226$ ), at Week 24 in $16 \mathrm{M}, 32 \mathrm{M}$, and Pooled groups (16 M: $P=0.0017 ; 32 \mathrm{M}: P=0.0039$; Pooled: $P=0.0039)$, and at Week 48 in $16 \mathrm{M}(16 \mathrm{M}: P=0.0392)$ in comparison with the HA group. (Fig. 4a, Additional file 2: Table S7). All groups had a significant increase in KSCRS symptoms score, patient satisfaction score, and functional activities from baseline to post-treatment at Week 4 to 48 (Additional file 2: Table S8, S9, $\mathrm{S} 10$ for detail $p$-value information). Moreover, the earlier significant symptoms relief were found at Week 2 


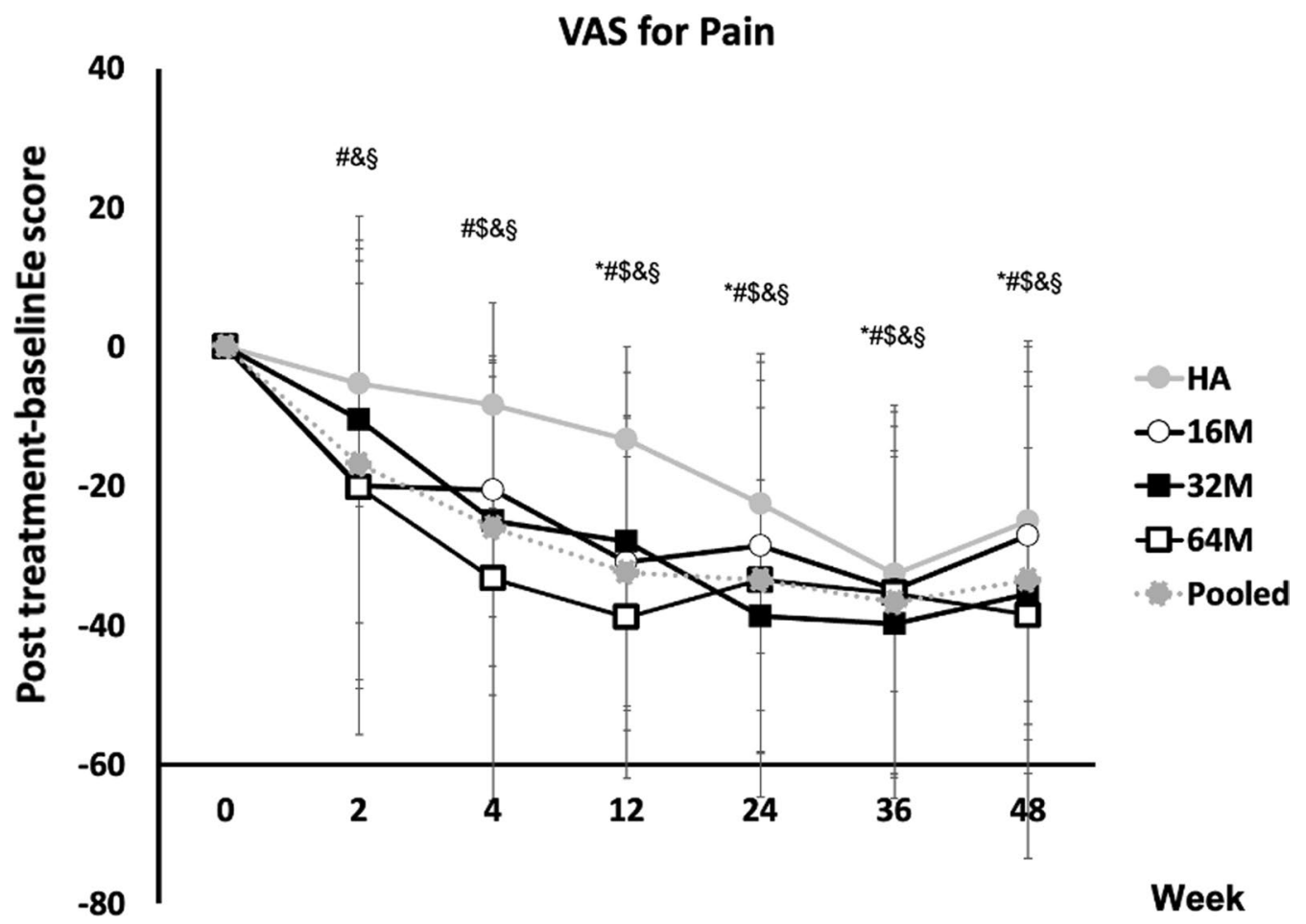

Fig. 3 Changes in VAS pain after intra-articular injection of ELIXCYTE ${ }^{\circledR}$ or HA at each time point. VAS pain scores are presented as mean \pm SD. $16 \mathrm{M}, 32 \mathrm{M}, 64 \mathrm{M}$, and $\mathrm{HA}=16,32,64$ million of ELIXCYTE ${ }^{\circledR}$, and hyaluronic acid, respectively. The signs of significance $={ }^{*}, \#, \$, \&, \S$ representing a $p$-value $<0.05$ between baseline and posttreatment score comparison for HA, $16 \mathrm{M}, 32 \mathrm{M}, 64 \mathrm{M}$, and Pooled group

in $16 \mathrm{M}, 32 \mathrm{M}$, and Pooled groups $(16 \mathrm{M}: P=0.0030$; 32 M: $P=0.0061$; Pooled: $P<0.0001)$. A significant difference of mean KSCRS Symptoms scores was observed at Week 12 in $16 \mathrm{M}, 64 \mathrm{M}$, and Pooled group (16 M: $P=0.0341 ; 64 \mathrm{M}: P=0.0080$; Pooled: $P=0.0300)$, and at Week 48 in $64 \mathrm{M}$ group in comparison with HA group $(P=0.0322)$ (Fig. 4b, Additional file 2: Table S8). The earlier significant improvements of patient satisfaction were found at Week 2 in $16 \mathrm{M}, 32 \mathrm{M}$, and Pooled groups (Additional file 2: Table S9, $16 \mathrm{M}: P=0.0359$; $32 \mathrm{M}: P=0.0022$; Pooled: $P=0.0014)$. The significant increases of mean KSCRS patient satisfaction scores were observed at Week 24 in $64 \mathrm{M}$ and Pooled groups (64 M: $P=0.0437$; Pooled: $P=0.0397$ ), and at Week 48 in $32 \mathrm{M}, 64 \mathrm{M}$, and Pooled groups in comparison with HA group (32 M: $P=0.0472$; $64 \mathrm{M}: P=0.0038$; Pooled: $P=0.0044$ ) (Fig. 4c, Additional file 2: Table S9). The HA group had significant increase in KSCRS functional activities score from baseline to post-treatment at Week 24 to 48 (Fig. 4d, Additional file 2: Table S10, $P=0.0133,0.0023,0.0252$ ), while the earlier changes in the individual ELIXCYTE ${ }^{\circledR}$ and Pooled groups were observed at Week 4 and continued to Week 48 (Additional file 2: Table S10 for detail $p$-value information).

\section{Discussion}

This present study aimed at evaluating the safety and efficacy of intra-articular ELIXCYTE ${ }^{\circledR}$ injection in patients with KOA. ELIXCYTE ${ }^{\circledR}$ for KOA treatment was shown to be effective, safe, and well-tolerated. The earlier onset and longer duration of effect mediated by ELIXCYTE ${ }^{\circledR}$ were observed compared to HA treatment and seem to show better outcomes in WOMAC scores, VAS score, and KSCRS scores.

ELIXCYTE ${ }^{\circledR}$ was shown to be safe, well-tolerated, and exhibited a comparable safety profile to the HA control. Although the allogeneic MSCs with poorly matched HLA had been reported to induce both innate and humoral responses, however, the response extent appears to be correlated with the expression level and the balance of both immune-activating antigens, such as major histocompatibility complex (MHC), and the immune-modifying cytokines, molecules and metabolites, like tumor necrosis factor-inducible gene 6 (TSG-6), galectin-1, prostaglandin E2 (PGE2), and indoleamine 2,3-dioxygenase (IDO) [23-27]. Regarding the immunogenicity results of our studies, the values of TNF- $\alpha, C D 4$, and CD8 values were normal or abnormal but NCS. Therefore, the safety profile in present studies was similar to 

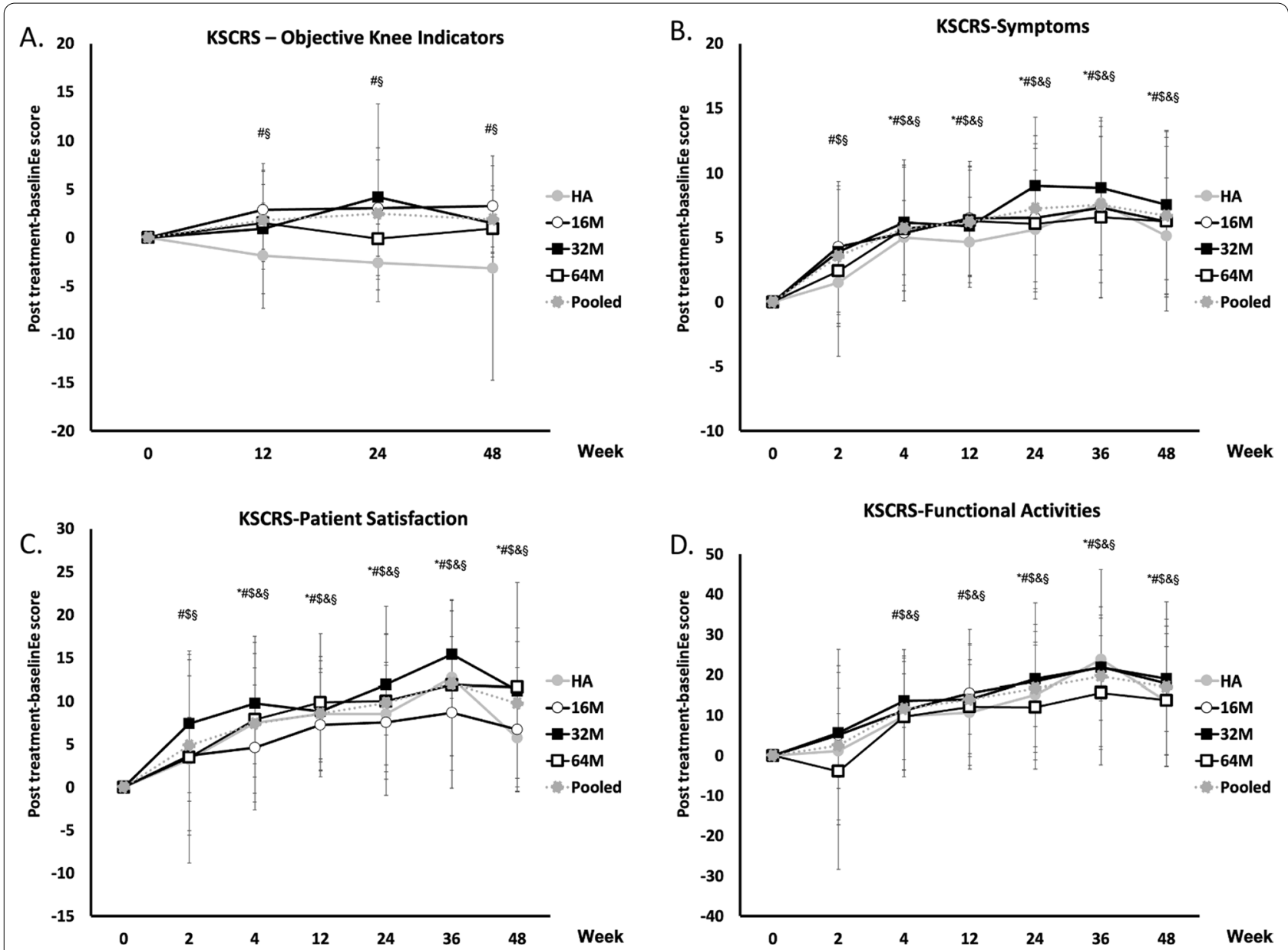

Fig. 4 Changes in KSCRS after intra-articular injection of ELIXCYTE ${ }^{\circledR}$ or HA at each time point. KSCRS objective knee indicators (a), symptoms (b), patient satisfaction (c), and functional activities (d) scores are presented as mean \pm SD. 16 M, $32 \mathrm{M}, 64 \mathrm{M}$, and HA=16, 32, 64 million of ELIXCYTE ${ }^{\circledR}$, and hyaluronic acid, respectively; The signs of significance $={ }^{*}, \#, \$, \&, \S$ representing a $p$-value $<0.05$ between baseline and posttreatment score comparison for HA, $16 \mathrm{M}, 32 \mathrm{M}, 64 \mathrm{M}$, and Pooled group

other human trials used adipose-derived stem cells for KOA treatment [28].

Compared to the HA group and $16 \mathrm{M}$ group, $32 \mathrm{M}$ and $64 \mathrm{M}$ groups seemed to have a larger percentage of general disorder and administration site conditions including pain and swelling at the injection site of the joint. The limited joint space, higher volume of injection (4 or $8 \mathrm{ml}$ ) may be the reason for those phenomena. Meanwhile, it could be assumed that a proportion of the cells injected into the joint space have not survived and this phenomenon was more pronounced with higher cell doses. Probably, such dead cells might trigger the inflammatory reaction in knee joint and thus cause pain [29]. Similar observation was found in Gupta's study, the adverse events, such as knee pain and swelling, were predominant in their higher-dose groups (50, 75, and 150 million cells) [30]. In another recent study, Vega et al. have conducted a study using IA injection of allogeneic bone marrow-derived MSCs (BMSC) (40 M cells suspended in $8 \mathrm{ml}$ of Ringer-Lactate). And the post-implantation pain was reported in $53 \%$ to $60 \%$ of patients in both the experimental and control groups [31]. Hence, pain and local swelling are the most common post-injection complication in patients after IA injection of MSCs.

The earlier onset and longer duration of pain and symptom control mediated by ELIXCYTE ${ }^{\circledR}$ were observed in this study compared to HA treatment. Allogeneic MSCs derived from adipose tissue and bone marrow have been described with the potential for more logistic convenience than autologous options. For example, the Stempeucel ${ }^{\circledR}$ which is an off-the-shelf ex vivo expanded bone marrow-derived allogeneic MSCs, was studied in phase II randomized trial with 60 patients. A non-statistically significant trend toward improvement in VAS and WOMAC scores compared with placebo was observed at 6 and 12 months [30]. However, MSCs from 
bone marrow appear to have high propensity to undergo chondrocyte hypertrophy and bone formation and thus may not be ideal for the repair of articular cartilage [3234]. Adipose tissue is an attractive source of therapeutic MSCs not only owing to less invasive procurement for harvest, but also due to higher MSC concentration than in bone marrow, lesser expression of MHC class I antigens, and greater replicative and secretory potential of MSC. Additionally, ADSCs has higher immunomodulatory capability that is an important property for treating of OA than BMSC $[35,36]$.

Although adipose tissue-derived stem cells have been reported to aid in cartilage repair as evidenced by arthroscopy and histological indications of hyalinelike cartilage regeneration [14]. It is still challenging to demonstrate a significant therapeutic improvement in cartilage structure by radiographic measures in clinical trials. Moreover, the relationship between the structural recovery of cartilage and the symptom relief of OA remains unclear. The effect of ELIXCYTE ${ }^{\circledR}$ on cartilage regeneration has also been evaluated by magnetic resonance imaging (MRI) in the present study. No cartilage regeneration or structural modification have been identified and that might be due to the small sample size, the insensitivity and variation of radiographic outcomes, and the effects covered by natural disease progression (Additional file 2: Table S11, S12). In addition, the efficacy of ELIXCYTE ${ }^{\circledR}$ was not in a dosage-dependent manner and the optimal dosage and application volume still need to be further explored. For achieving effective and durable therapeutic effect, administration of sufficient amount of MSCs is critical. Repeated dosing of MSCs has demonstrated a superior clinical outcome compared to single dose in previous study [37]. Reaching sufficient cell numbers by giving smaller volumes of repeated doses may be one of the strategies for designing treatments in the further development of ELIXCYTE ${ }^{\circledR}$.

\section{Conclusions}

In summary, the safety and therapeutic efficacy of allogenic ADSC therapy for treating KOA patients was reported. All ELIXCYTE ${ }^{\circledR}$ groups presented a comparable safety profile to HA along with an earlier onset and longer duration of the effectiveness compared to the HA. The administration of ELIXCYTE ${ }^{\circledR}$ is low risk and these results support the continued development of ELIXCYTE ${ }^{\circledR}$.

\section{Abbreviations}

ADSCs: Adipose-derived stem cells; IA: Intra-articular; HA: Hyaluronic acid; WOMAC: Western Ontario and McMaster Universities Osteoarthritis Index; AEs: Adverse events; SAEs: Serious adverse events; SUSAR: Suspected unexpected serious adverse reaction; VAS: Visual analog scale; KSCRS: Knee Society Clinical Rating System; KOA: Knee osteoarthritis; YLDs: Years lived with disability;
NSAID: Nonsteroidal anti-inflammatory drug; DMOAD: Disease-modifying osteoarthritis drug; MSCs: Mesenchymal stem cells; SVF: Stromal vascular fraction; CRO: Contract research organization; M: Million; ACR: American College of Rheumatology; HIV: Human immunodeficiency virus; BMI: Body mass index; WBC: White blood cells; RBC: Red blood cells; BUN: Blood urea nitrogen; TNF-a: Tumor necrosis factor alpha; ITT: Intent-to-treat; KL: Kellgren-Lawrence; TEAE: Treatment-emergent adverse event; SOC: System organ class; NCS: No clinically significant; AST: Aspartate aminotransferase; MHC: Major histocompatibility complex; TSG-6: Tumor necrosis factor-inducible gene 6, galectin-1; PGE2: Prostaglandin E2; IDO: Indoleamine 2,3-dioxygenase; BMSCs: Bone marrowderived MSCs; MRI: Magnetic resonance imaging.

\section{Supplementary Information}

The online version contains supplementary material available at https://doi. org/10.1186/s13287-021-02631-z.

Additional file 1: Hematology and biochemistry data from patients in trial.

Additional file 2: Supplementary safety and efficacy data of ELIXCYTE ${ }^{\circledR}$ including Table S1-S12.

\section{Acknowledgements}

We express our appreciation to the two institutions for sponsorship, UnicoCell BioMed and the $\mathrm{A}^{+}$Industrial Innovative R\&D Program, Ministry of Economic Affairs, R.O.C. We would also like to express our sincere gratitude to all the patients and the medical professionals who participated in this trial.

\section{Authors' contributions}

All authors made substantial contributions to all of the following: (1) the conception and design of the study, or acquisition of data, or analysis and interpretation of data, (2) drafting the article or revising it critically for important intellectual content, (3) final approval of the version to be submitted.

\section{Funding}

This study was funded by UnicoCell BioMed Co. Ltd. and the $\mathrm{A}^{+}$Industrial Innovative R\&D Program, Ministry of Economic Affairs, R.O.C (Grant No. 105-EC-17-A-22-15-0007).

\section{Availability of data and materials}

The datasets used or analyzed during the current study are available from the corresponding author on reasonable request.

\section{Declarations}

Ethics approval and consent to participate

The protocol of the study was approved by the Institutional Review Board to the tenets of the Declaration of Helsinki. There were two study sites in this trial, including Linkou Chang Gung Memorial Hospital and Taipei Veterans General Hospital in Taiwan. The official ethical approval numbers of the study were 104-1182A (CGMF-IRB) and 2017-04-005A (VGHTPE-IRB). The study was registered at http://ClinicalTrials.gov (NCT02784964).

\section{Consent for publication}

Not applicable.

\section{Competing interests}

The authors declare that they have no competing interests.

\section{Author details}

${ }^{1}$ Division of Joint Reconstruction, Department of Orthopedics and Traumatology, Taipei Veterans General Hospital, Taipei, Taiwan. ${ }^{2}$ Department of Surgery, School of Medicine, National Yang Ming Chiao Tung University, Taipei, Taiwan. ${ }^{3}$ Division of Joint Reconstruction, Department of Orthopedic Surgery, Chang Gung Memorial Hospital, Linkou, Taiwan. ${ }^{4}$ Bone and Joint Research Center, Chang Gung Memorial Hospital, Linkou, Taiwan. ${ }^{5}$ College of Medicine, Chang Gung University, Taoyuan, Taiwan. ${ }^{6}$ Department of Medical Imaging and Intervention, Chang Gung Memorial Hospital, Linkou, Taiwan. ${ }^{7}$ Department 
of Radiology, Taipei Veterans General Hospital, Taipei, Taiwan. ${ }^{8}$ Department of Radiology, School of Medicine, National Yang Ming Chiao Tung University, Taipei, Taiwan. ${ }^{9}$ Craniofacial Research Center, Department of Medical Research, Department of Plastic and Reconstructive Surgery and Department of Craniofacial Orthodontics, Chang Gung Memorial Hospital, Taoyuan, Taiwan. ${ }^{10}$ Department of Chemical and Materials Engineering, College of Engineering, Chang Gung University, Taoyuan, Taiwan. ${ }^{11}$ UnicoCell BioMed Co. Ltd., Taipei, Taiwan.

Received: 1 August 2021 Accepted: 4 October 2021

Published online: 30 October 2021

\section{References}

1. Hunter DJ, March L, Chew M. Osteoarthritis in 2020 and beyond: a Lancet Commission. Lancet. 2020;396(10264):1711-2.

2. Ghouri A, Conaghan PG. Update on novel pharmacological therapies for osteoarthritis. Therapeutic advances in musculoskeletal disease. 2019;11:1759720x19864492.

3. Hunter DJ, Bierma-Zeinstra S. Osteoarthritis. Lancet (London, England). 2019;393(10182):1745-59.

4. Migliore A, Paoletta M, Moretti A, Liguori S, Iolascon G. The perspectives of intra-articular therapy in the management of osteoarthritis. Exp Opin Drug Deliv. 2020;17(9):1213-26.

5. Rezuş E, Burlui A, Cardoneanu A, Macovei LA, Tamba BI, Rezuş C. From pathogenesis to therapy in knee osteoarthritis: bench-to-bedside. Int J Mol Sci. 2021;22(5):2697.

6. Harrell CR, Markovic BS, Fellabaum C, Arsenijevic A, Volarevic V. Mesenchymal stem cell-based therapy of osteoarthritis: current knowledge and future perspectives. Biomed Pharmacother Biomed Pharmacother. 2019;109:2318-26.

7. Pers YM, Ruiz M, Noël D, Jorgensen C. Mesenchymal stem cells for the management of inflammation in osteoarthritis: state of the art and perspectives. Osteoarthritis Cartilage. 2015;23(11):2027-35.

8. Barry F, Murphy M. Mesenchymal stem cells in joint disease and repair. Nat Rev Rheumatol. 2013;9(10):584-94.

9. Black LL, Gaynor J, Adams C, Dhupa S, Sams AE, Taylor R, et al. Effect of intraarticular injection of autologous adipose-derived mesenchymal stem and regenerative cells on clinical signs of chronic osteoarthritis of the elbow joint in dogs. Vet Therap Res Appl Vet Med. 2008;9(3):192-200.

10. Lee KB, Hui JH, Song IC, Ardany L, Lee EH. Injectable mesenchymal stem cell therapy for large cartilage defects-a porcine model. Stem Cells. 2007;25(11):2964-71.

11. Murphy JM, Fink DJ, Hunziker EB, Barry FP. Stem cell therapy in a caprine model of osteoarthritis. Arthritis Rheum. 2003;48(12):3464-74.

12. van Buul GM, Siebelt M, Leijs MJ, Bos PK, Waarsing JH, Kops N, et al. Mesenchymal stem cells reduce pain but not degenerative changes in a monoiodoacetate rat model of osteoarthritis. J Orthop Res. 2014;32(9):1167-74.

13. Centeno CJ, Schultz JR, Cheever M, Robinson B, Freeman M, Marasco W. Safety and complications reporting on the re-implantation of cultureexpanded mesenchymal stem cells using autologous platelet lysate technique. Curr Stem Cell Res Ther. 2010;5(1):81-93.

14. Jo CH, Lee YG, Shin WH, Kim H, Chai JW, Jeong EC, et al. Intra-articular injection of mesenchymal stem cells for the treatment of osteoarthritis of the knee: a proof-of-concept clinical trial. Stem Cells. 2014;32(5):1254-66.

15. Vega A, Martín-Ferrero MA, Del Canto F, Alberca M, García V, Munar A, et al. Treatment of knee osteoarthritis with allogeneic bone marrow mesenchymal stem cells: a randomized controlled trial. Transplantation. 2015;99(8):1681-90.

16. Centeno CJ, Busse D, Kisiday J, Keohan C, Freeman M, Karli D. Increased knee cartilage volume in degenerative joint disease using percutaneously implanted, autologous mesenchymal stem cells. Pain Phys. 2008;11(3):343-53.

17. Song Y, Du H, Dai C, Zhang L, Li S, Hunter DJ, et al. Human adipose-derived mesenchymal stem cells for osteoarthritis: a pilot study with long-term follow-up and repeated injections. Regen Med. 2018;13(3):295-307.

18. Lu L, Dai C, Zhang Z, Du H, Li S, Ye P, et al. Treatment of knee osteoarthritis with intra-articular injection of autologous adipose-derived mesenchymal progenitor cells: a prospective, randomized, double-blind, active-controlled, phase Ilb clinical trial. Stem Cell Res Ther. 2019;10(1):143.
19. Lee WS, Kim HJ, Kim Kl, Kim GB, Jin W. Intra-articular injection of autologous adipose tissue-derived mesenchymal stem cells for the treatment of knee osteoarthritis: a phase $\mathrm{llb}$, randomized, placebo-controlled clinical trial. Stem Cells Transl Med. 2019;8(6):504-11.

20. Freitag J, Bates D, Wickham J, Shah K, Huguenin L, Tenen A, et al. Adiposederived mesenchymal stem cell therapy in the treatment of knee osteoarthritis: a randomized controlled trial. Regen Med. 2019;14(3):213-30.

21. Xinxin Han BY, Fagui Zou, Jianbo Sun. Clinical therapeutic efficacy of mesenchymal stem cells derived from adipose or bone marrow for knee osteoarthritis: a meta-analysis of randomized controlled trials. J Comp Eff Res. 2020.

22. Zhao X, Ruan J, Tang H, Li J, Shi Y, Li M, et al. Multi-compositional MRI evaluation of repair cartilage in knee osteoarthritis with treatment of allogeneic human adipose-derived mesenchymal progenitor cells. Stem Cell Res Ther. 2019;10(1):308.

23. Caplan H, Olson SD, Kumar A, George M, Prabhakara KS, Wenzel P, et al. Mesenchymal stromal cell therapeutic delivery: translational challenges to clinical application. 2019;10(1645).

24. Ankrum JA, Ong JF, Karp JM. Mesenchymal stem cells: immune evasive, not immune privileged. Nat Biotechnol. 2014;32(3):252-60.

25. Consentius C, Reinke P, Volk HD. Immunogenicity of allogeneic mesenchymal stromal cells: what has been seen in vitro and in vivo? Regen Med. 2015;10(3):305-15.

26. Berglund AK, Fortier LA, Antczak DF, Schnabel LV. Immunoprivileged no more: measuring the immunogenicity of allogeneic adult mesenchymal stem cells. Stem Cell Res Therapy. 2017;8(1):288.

27. Lohan P, Treacy O, Griffin MD, Ritter T, Ryan AE. Anti-donor immune responses elicited by allogeneic mesenchymal stem cells and their extracellular vesicles: are we still learning? Front Immunol. 2017;8:1626.

28. Ip HL, Nath DK, Sawleh SH, Kabir MH, Jahan N. Regenerative medicine for knee osteoarthritis - the efficacy and safety of intra-articular platelet-rich plasma and mesenchymal stem cells injections: a literature review. Cureus. 2020;12(9):e10575.

29. Rock KL, Kono H. The inflammatory response to cell death. Annu Rev Pathol. 2008;3:99-126.

30. Gupta PK, Chullikana A, Rengasamy M, Shetty N, Pandey V, Agarwal V, et al. Efficacy and safety of adult human bone marrow-derived, cultured, pooled, allogeneic mesenchymal stromal cells (Stempeucel(R)): preclinical and clinical trial in osteoarthritis of the knee joint. Arthritis Res Ther. 2016;18(1):301.

31. Vega A, Martin-Ferrero MA, Del Canto F, Alberca M, Garcia V, Munar A, et al. Treatment of knee osteoarthritis with allogeneic bone marrow mesenchymal stem cells: a randomized controlled trial. Transplantation. 2015;99(8):1681-90.

32. Vinardell T, Sheehy EJ, Buckley CT, Kelly DJ. A comparison of the functionality and in vivo phenotypic stability of cartilaginous tissues engineered from different stem cell sources. Tissue Eng Part A. 2012;18(11-12):1161-70.

33. Scotti C, Tonnarelli B, Papadimitropoulos A, Scherberich A, Schaeren S, Schauerte A, et al. Recapitulation of endochondral bone formation using human adult mesenchymal stem cells as a paradigm for developmental engineering. Proc Natl Acad Sci USA. 2010;107(16):7251-6.

34. De Bari C, Roelofs AJ. Stem cell-based therapeutic strategies for cartilage defects and osteoarthritis. Curr Opin Pharmacol. 2018;40:74-80.

35. Strioga M, Viswanathan S, Darinskas A, Slaby O, Michalek J. Same or not the same? Comparison of adipose tissue-derived versus bone marrow-derived mesenchymal stem and stromal cells. Stem Cells Dev. 2012;21(14):2724-52.

36. Melief SM, Zwaginga JJ, Fibbe WE, Roelofs H. Adipose tissue-derived multipotent stromal cells have a higher immunomodulatory capacity than their bone marrow-derived counterparts. Stem Cells Transl Med. 2013;2(6):455-63.

37. Matas J, Orrego M, Amenabar D, Infante C, Tapia-Limonchi R, Cadiz MI, et al. Umbilical cord-derived mesenchymal stromal cells (MSCs) for knee osteoarthritis: repeated MSC dosing is superior to a single MSC dose and to hyaluronic acid in a controlled randomized phase I/II trial. Stem Cells Transl Med. 2019;8(3):215-24.

\section{Publisher's Note}

Springer Nature remains neutral with regard to jurisdictional claims in published maps and institutional affiliations. 